\title{
Fast piezoelectric scanning MEMS mirror for 1D ion addressing
}

\author{
Paul Janin, Ralf Bauer, Paul Griffin, Erling Riis, and Deepak Uttamchandani \\ University of Strathclyde, Glasgow, United Kingdom \\ paul.janin@strath.ac.uk
}

\begin{abstract}
We present a small-scale piezoelectric MEMS micromirror, with resonant frequencies above $300 \mathrm{kHz}$ for $1 D$ scanning. The device is intended for higher frequency operation by reducing the scale of existing designs, and was fabricated using a multi-user silicon-on-insulator process. The performance of the mirror for addressing points along one axis was demonstrated using a free-space optics experimental setup.
\end{abstract}

Index Terms-optical MEMS, piezoelectric, scanning mirror, high frequency

\section{INTRODUCTION}

MEMS resonant tilting mirrors have been widely used in scanning applications, most notably for projection displays. The demand for higher-resolution displays has driven an interest in faster, more efficient scanners, and numerous innovative designs have been presented in recent years [1]. Actuation methods for such devices include electrothermal, electrostatic and piezoelectric actuators. Novel applications looking at uses of MEMS scanners in ultrafast X-ray manipulation [2] or for quantum optics applications, such as addressing of trapped atomic particles [3], require even faster repetition rate scanners due to the typically short operation time scales.

In this paper we present a high frequency scanning micromirror using cost-effective single crystal silicon multi-user fabrication with piezoelectric actuation for beam steering. The device is designed to address atomic particle traps. We also demonstrate the device capability to steer laser pulses to arbitrary points aligned perpendicularly to the optical axis.

\section{DEVICE DESIGN}

The developed device is a silicon on insulator resonant tilting mirror with a double gimbal frame design. The device geometry is based on the one presented in [4], with a significant reduction in scale and corresponding frequency increase. The mirror plate has a $200 \mu \mathrm{m}$ diameter with a $10 \mu \mathrm{m}$ thick silicon device layer, surrounded by a $20 \mu \mathrm{m}$ circular frame and a $100 \mu \mathrm{m}$ wide elliptic frame. The whole device is fully back-side released through a $400 \mu \mathrm{m}$ handle wafer. A thin $(500 \mathrm{~nm})$ film of aluminum nitride is deposited in four separate sections on the outer frame, forming the mirror actuators. This piezoelectric layer is covered with $1.0 \mu \mathrm{m}$ of aluminum, forming the top electrode, with the device layer acting as common ground.

P.J. was supported by NPL through a NPL Research Studentship and R.B. by the Royal Academy of Engineering under the Engineering for Development Research Fellowship scheme (RF1516 \15\8)
Each actuator is electrically connected to an aluminum pad, insulated from the silicon base by a layer of silicon oxide. Piezoelectric mirror actuation is achieved through voltage control of each aluminum electrode. A schematic layout of the device is shown on Fig. 1.

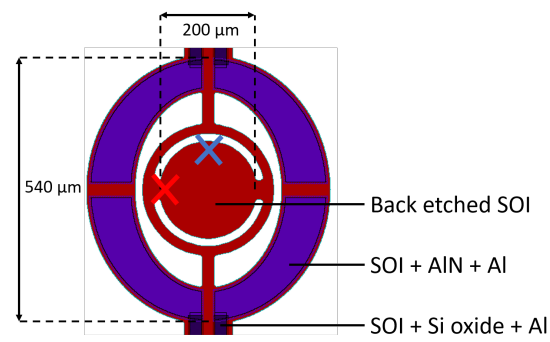

Fig. 1. Layout schematic of the micromirror. Crosses indicate the vibrometer measurement points used for Fig. 2, with colors respective to plotting lines.

\section{DEVICE CHARACTERIZATION}

The device mechanical structure was simulated using the finite-element simulation software COMSOL Multiphysics 5.3. The resulting displacement mode shapes are shown as insets in Fig. 2, with simulated frequencies of $158 \mathrm{kHz}$, $183.1 \mathrm{kHz}$ and $360.2 \mathrm{kHz}$ for a mirror piston, tip and tilt movement respectively. The design was fabricated using MEMSCAP's commercial PiezoMUMPS process. After fabrication, the device was characterized using a laser doppler vibrometer (Polytec OFV 3001), providing a single point velocity reading of the device during actuation. Specific mode shapes of interest were then investigated using a Veeco NT1100 dynamic optical profiler, following the characterization process detailed in [5]. Fig. 2 shows the vibrometry response, converted to a mechanical scan angle, at two points on the mirror plate using a $20 \mathrm{~V}_{p p}+0 \mathrm{~V}_{d c}$ sine-wave actuation of a single actuator. It should be noted that the response was measured on a single device sampled from a batch of 17 fabricated chips. A variance in resonance frequencies of approximately $1-5 \%$ was observed among different chips of the same batch. This is attributed to fabrication tolerances such as uneven layer thickness and material stress across the wafers during the deposition process. The chosen operation frequency for 1D addressing and the subsequent experimental results was the third mode at approximately $335 \mathrm{kHz}$.

White-light interferometry provides measurements to confirm the mode shapes predicted by simulation, and analyze 
any dynamic mirror surface deformation. For the tipping and tilt modes, the mirror exhibits mainly astigmacy trefoil aberration, with out-of plane displacement of less than $150 \mathrm{~nm}$ for $329.4 \mathrm{kHz}$ (pictured in Fig. 3), $50 \mathrm{~nm}$ for $150.5 \mathrm{kHz}$ and $60 \mathrm{~nm}$ for $173.2 \mathrm{kHz}$ at $10 \mathrm{~V}_{p p}$ actuation. This relates to a low surface deformation between $\lambda / 3$ and $\lambda / 4$ for visible wavelengths.

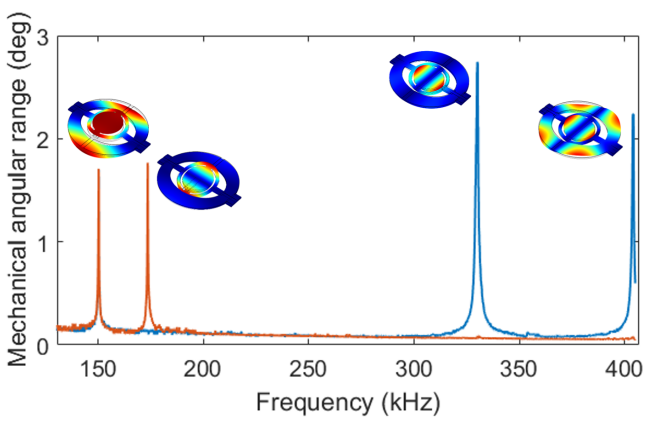

Fig. 2. Measured optical tilt angle frequency response for $20 \mathrm{~V}$ sinewave actuation. Insets are the simulated mode shapes at the corresponding Eigenfrequencies.

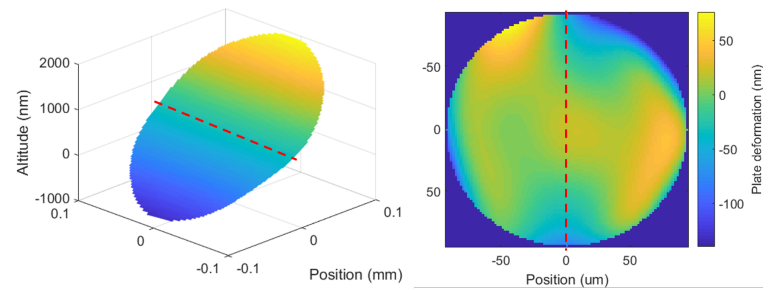

Fig. 3. Left : plate surface profile at maximum tilt angle for $10 \mathrm{~V}_{p p}+5 \mathrm{~V}_{d c}$ actuation at $329.4 \mathrm{kHz}$. Right : plate dynamic surface deformation after correction for tilt and Zernike polynomial smoothing.

\section{OpticAl SETUP FOR 1D ADDRESSING}

In order to further characterize the device performances as a scanner for applications such as atomic particle traps, a freespace optics setup was created using the experimental layout shown in Fig. 4. Laser pulses are synchronized with the piezo actuation frequency and are produced by a red laser diode (Thorlabs L638P700M) driven by a picosecond pulse driver (Analog modules model 766), triggered by a two channel signal generator (Rigol DG1022) handling the synchronization between the MEMS actuation signal and the pulse trigger TTL signal. The laser output is coupled through a single mode fiber to ensure a $\mathrm{TEM}_{00}$ Gaussian beam shape on the mirror. The collimated beam is focused on the mirror plate and then re-focused on a CMOS camera (Thorlabs DCC1545M). The $f=120 \mathrm{~mm}$ and $f^{\prime}=80 \mathrm{~mm}$ focusing lenses are positioned with $\delta \approx 12 \mathrm{~mm}$ so that a telecentric system is achieved after the second lens.

The pulse positions observed on the camera are shown in Fig. 5 for a MEMS actuation voltage of $40 \mathrm{~V}_{p p}$ and frequency of $341.6 \mathrm{kHz}$, with each image the sum of several thousand pulses. The spot size on the detector is approximately
$350 \mu \mathrm{m}$. The observed spacing between the two pulses can be controlled continuously by changing the pulse trigger phase relative to the actuation signal, with the three representative phases shown relating to pulsing at the mirror rest position $\left(0^{\circ}\right)$, as well as $45^{\circ}$ and $90^{\circ}$ from it.

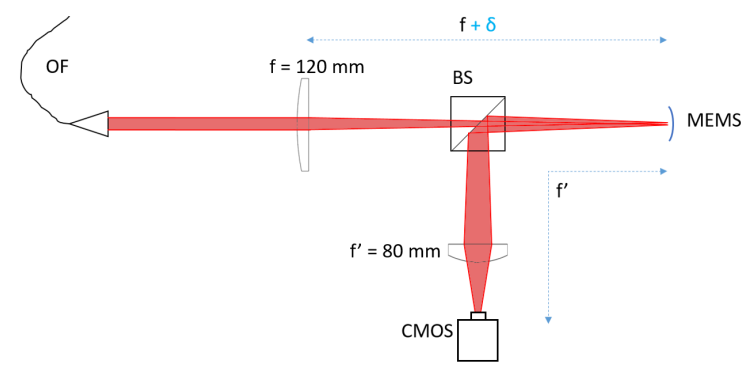

Fig. 4. Optical setup for $1 \mathrm{D}$ addressing demonstration. "OF" is the single mode fiber for light delivery, "BS" is a 50/50 beam splitter.

\section{COnClusions}

We have demonstrated a fast $1 \mathrm{D}$ addressing of an array of points using a piezoelectric tilting mirror at over $300 \mathrm{kHz}$. The use of the device's mechanical resonance allows for a high frequency operation while retaining a satisfactory (more than $5^{\circ}$ ) deflection range for the intended trapped atomic particle addressing. In our future work, this device will be used as part of a $2 \mathrm{D}$ beam steering system for small scale trapped atom addressing.

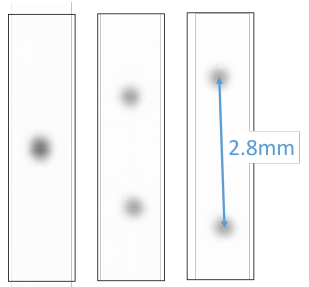

Fig. 5. Spatial pulse profiles as seen on the CMOS camera, for an actuation frequency of $341.6 \mathrm{kHz}$ and a laser pulse frequency of $683.2 \mathrm{kHz}$. From left to right : $0^{\circ}, 45^{\circ}$ and $90^{\circ}$ actuation phase shift relative to the mirror movement period.

\section{REFERENCES}

[1] S. T. Holmstrom, U. Baran, and H. Urey, "MEMS laser scanners: a review,' Journal of Microelectromechanical Systems, vol. 23, no. 2, pp. 259-275, 2014.

[2] P. Chen, I. W. Jung, D. A. Walko, Z. Li, Y. Gao, G. K. Shenoy, D. López, and J. Wang, "Ultrafast photonic micro-systems to manipulate hard Xrays at 300 picoseconds," Nature Communications, vol. 10, no. 1, p. 1158, 2019.

[3] C. Knoernschild, C. Kim, F. P. Lu, and J. Kim, "Multiplexed broadband beam steering system utilizing high speed MEMS mirrors," Optics Express, vol. 17, no. 9, pp. 7233-7244, 2009.

[4] U. Baran, D. Brown, S. Holmstrom, D. Balma, W. O. Davis, P. Muralt, and H. Urey, "Resonant PZT MEMS scanner for high-resolution displays," Journal of Microelectromechanical Systems, vol. 21, no. 6, pp. 1303-1310, 2012.

[5] P. Janin, R. Bauer, P. Griffin, E. Riis, and D. Uttamchandani, "Characterization of a Fast Piezoelectric Varifocal MEMS Mirror," International Conference on Optical MEMS and Nanophotonics, pp. 155-556, 2018. 